\title{
Uncontrolled asthma from childhood to young adulthood associates with airflow obstruction
}

\author{
Ida Mogensen $\mathbb{B}^{1}$, Jenny Hallberg ${ }^{1,2}$, Sandra Ekström ${ }^{3,4}$, Anna Bergström ${ }^{3}$, Erik Melén $\mathbb{B}^{1,2}$ and \\ Inger Kull ${ }^{1,2}$
}

${ }^{1}$ Dept of Clinical Science and Education Södersjukhuset, Karolinska Institute, Stockholm, Sweden. ${ }^{2}$ Sachs' Children and Youth Hospital, Södersjukhuset, Stockholm, Sweden. ${ }^{3}$ Institute of Environmental Medicine, Karolinska Institute, Stockholm, Sweden. ${ }^{4}$ Center for Occupational and Environmental Medicine, Region Stockholm, Stockholm, Sweden.

Corresponding author: Ida Mogensen (ida.mogensen@ki.se)

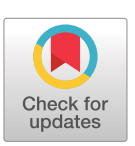

This version is distributed under the terms of the Creative Commons Attribution Non-Commercial Licence 4.0. For commercial reproduction rights and permissions contact permissions@ersnet.org

This article has supplementary material available from openres.ersjournals.com

Received: 13 March 2021 Accepted: 1 June 2021

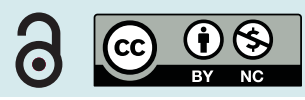

Shareable abstract (@ERSpublications)

Uncontrolled asthma from 8 to 24 years of age is associated with a lower overall FEV $/$ FVC $z$-score. Intensified treatment of symptomatic asthma, especially asthma with elevated $F_{\text {ENO }}$ and in females, could have important implications for future lung health. https://bit.ly/3pHkleN

Cite this article as: Mogensen I, Hallberg J, Ekström S, et al. Uncontrolled asthma from childhood to young adulthood associates with airflow obstruction. ERJ Open Res 2021; 7: 00179-2021 [DOI: 10.1183/ 23120541.00179-2021].

\section{Abstract}

Introduction Lung function development from childhood to young adulthood is important for lung health later in life. We investigated the association between asthma control and lung function from 8 to 24 years of age.

Methods A total of 668 participants from the population-based BAMSE cohort study, with persistent or incidental asthma and between 8 and 24 years of age, were included. Asthma was defined as controlled or uncontrolled at each examination based on the Global Initiative for Asthma (GINA) criteria. Dynamic spirometry was performed at 8, 16 and 24 years of age. Associations between uncontrolled asthma and prebronchodilation forced expiratory volume in $1 \mathrm{~s}\left(\mathrm{FEV}_{1}\right)$, forced vital capacity (FVC) and $\mathrm{FEV}_{1} / \mathrm{FVC}$ ratio were evaluated with a generalised estimating equation model, as overall associations and at each examination. Unadjusted and adjusted (for sex, current asthma, allergic sensitisation, body mass index, smoking, smoke exposure, inhaled corticosteroid use) analyses were done; and were thereafter stratified by sex, elevated blood eosinophils $\left(\geqslant 0.3 \times 10^{9}\right.$ cells $\left.\cdot \mu \mathrm{L}^{-1}\right)$, elevated $F_{\mathrm{ENO}}(\geqslant 25 \mathrm{ppb})$, allergic sensitisation and ever/never smoking.

Results Uncontrolled asthma was associated with a lower overall $\mathrm{FEV}_{1} / \mathrm{FVC}$ z-score from 8 to 24 years of age (adjusted regression coefficient -0.11 ; $95 \%$ CI $(-0.20$ to -0.02 ; $p=0.016)$. After stratification, this association was primarily seen among females (adjusted regression coefficient -0.170 ; 95\% CI ( -0.298 to $-0.044 ; \mathrm{p}=0.009$ ) and participants with elevated $F_{\mathrm{ENO}}$ (regression coefficient $-0.207 ; 95 \% \mathrm{CI}-0.342$ to -0.073 ; $=0.002$ ), in contrast to males and participants with normal $F_{\text {ENO. }}$.

Conclusion Uncontrolled asthma is associated with airflow obstruction from childhood to young adulthood. This highlights the importance of active management of asthma during growth.

\section{Introduction}

Low lung function and airflow obstruction in adolescence are risk factors for respiratory conditions such as fixed airflow obstruction and chronic obstructive lung disease later in life, as well as for early death [1-3]. The peak attained lung function reached around the age of 25-30 [4] is important for the risk of developing a symptomatic lung function impairment at an older age. Lung function development tends to follow an almost predetermined trajectory depending on both pre- and early postnatal factors [2]. However, even if the ability to reach a higher trajectory seems to be limited, environmental factors can have a detrimental influence on the growth gain in lung function during childhood and adolescence [5-8]. The period from childhood to adulthood, i.e. transition, is in many ways a challenging time, even more so for individuals with a chronic disease. The referral from child to adult medicine is a potential risk for treatment gaps and to "get lost" in between children and adult healthcare [9, 10]. 
Asthma is a known risk factor for airflow obstruction and a lower maximal lung function plateau [6, 11]. Asthma is an inflammatory disease, and the inflammatory activity is believed to be the main factor leading to the adverse effects of asthma: impaired lung growth, asthma symptoms and exacerbations. Presence of eosinophil inflammation has been linked to impaired lung function development [12, 13] as well as fixed airflow obstruction [14]. Further, the presence of asthma exacerbations has been found to be associated with a worse lung function development $[15,16]$, with the underlying hypothesis that inflammatory bursts are leading to airway damage. Indeed, corticosteroid treatment has been linked to better outcomes in some studies [17, 18], possibly due to the decreased risk of asthma attacks [16] or attenuated inflammation. However, many asthma patients in younger age have mild asthma [19] and how less severe events are associated with lung function development is therefore important.

There are known gender differences in prevalence and incidence of asthma during childhood and adolescence. Males have been shown to have a rather stable prevalence during childhood and adolescence. In comparison, females have a lower prevalence during childhood and marked increase during adolescence, and the phenotype seems to be more heterogenous in females compared to males [20, 21].

The aim of this study was to investigate uncontrolled asthma compared to controlled asthma and the association with lung function measured with dynamic spirometry at age 8, 16 and 24 years. Stratification for sex was done due to the gender differences in asthma morbidity from childhood to early adulthood, and for elevated inflammatory markers and allergy for a better understanding of underlying pathology. The hypothesis was that uncontrolled asthma is associated with impaired lung function and airflow obstruction from childhood to early adulthood.

Methods

Study population

The included population comes from the BAMSE (Children, Allergy, Milieu, Stockholm, Epidemiology) cohort, a population-based birth cohort from Stockholm, Sweden, initiated in 1994, with the aim of studying the origins and characteristics of asthma and allergic diseases. The initial cohort consisted of 4089 individuals [22]. The group has been followed for 24 years and examined with questionnaires (age 0 , 1,2 and 12) covering exposures and allergic and asthmatic symptoms, and questionnaires and clinical examinations at approximately 4, 8, 16 and 24 years [23].

For this investigation, the period from 8 to 24 years of age was included, covering the three examinations at 8,16 and 24 years of age. Participants were included if they had completed at least one spirometry and fulfilled the criteria for asthma at one or more examinations (figure 1). Asthma was defined as present at a given examination if the participant fulfilled at least two of the following three criteria: physician-diagnosed asthma (ever), wheeze or raspy breathing in the previous 12 months and/or treatment for asthma during the previous 12 months [24].

\section{Questionnaire data}

Questionnaires were completed by parents at 8 and 16 years, and by participants at 16 and 24 years. Symptoms were assessed at each examination through a questionnaire covering the previous year and built on a modified GINA asthma control score [25]. In short, one point was given each for wheezy or raspy breathing: nightly

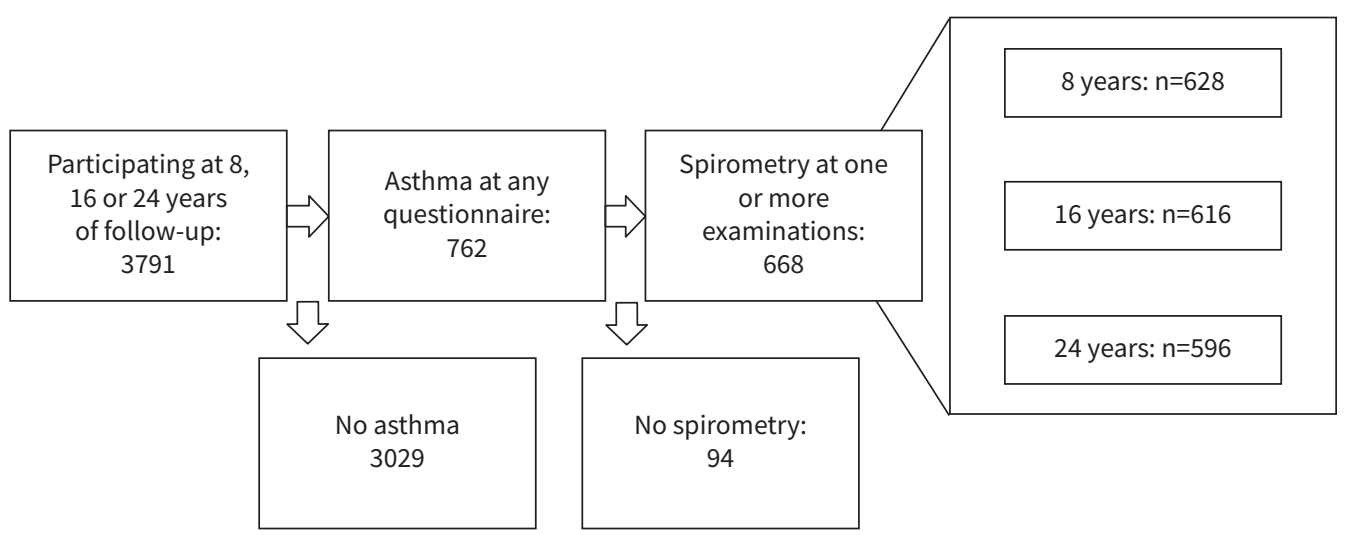

FIGURE 1 Flow chart of included individuals. 
(monthly); daytime (monthly); limiting daily activities (last year); need of short-acting bronchodilator (SABA) rescue treatment ( $\geqslant 2$ times/week) and asthma exacerbations (last year). Controlled asthma was defined as an asthma control score at 0 points, and uncontrolled $>0$ points (Supplementary Table 1).

Environmental smoke exposure was considered present when one parent reported smoking (at 8 and 16 years) or at 24 years when the participant reported exposure to indoor smoking at least once a week. A participant was regarded to be a smoker if reporting smoking daily or occasionally (16 and 24 years); all participants were considered nonsmokers at 8 years.

Inhaled corticosteroid (ICS) use was self-reported and categorised into never users, intermittent users using ICS for shorter periods and persistent users continuously using ICS.

Body mass index (BMI) was based on information on height and weight measured at the clinical examinations and calculated and categorised into under/normal weight (BMI <25), overweight (BMI 25-30) and obese $(\mathrm{BMI} \geqslant 30)$. At 8 and 16 years, the International Obesity Task Force (IOTF) cut-offs [26] were used.

\section{Spirometry}

At 8 years a Sensormedics 2200 Pulmonary Function Analyzer (Sensormedics, Anaheim, CA, USA), at 16 years a Jaeger MasterScreen-IOS system (Carefusion Technologies, San Diego, CA, USA) and at 24 years a Vyaire Vyntus system (Vyaire Medical, IL, USA) was used to measure dynamic pre-bronchodilatory spirometry [3]. Lung function was converted to z-score according to the Global Lung Initiative (GLI) reference values [27].

\section{Laboratory measures}

Allergic sensitisation was measured with Phadiatop mix (Thermo Fischer Scientific, Uppsala, Sweden), measuring IgE against mould, birch, cat, dog, horse, timothy, mugwort or house dust mite and considered positive with a value $\geqslant 0.35 \mathrm{kU} \cdot \mathrm{L}^{-1}[23]$.

$F_{\text {ENO }}$ was measured with an Eco Medics instrument system (Eco Medics AG, Dürnten, Switzerland) at the 16-year examination and with NIOX VERO (Circassia, Oxford, UK) at the 24-year examination, at a velocity of $50 \mathrm{~mL} \cdot \mathrm{s}^{-1}$ according to the ATS/ERS guidelines [28]. $F_{\text {ENO }}$ was considered elevated for values $\geqslant 25$ ppb [29].

Blood eosinophils were measured with a Sysmex XE (Sysmex, Kobe, Japan) at 16 years and a XN 9000 at 24 years at a precision of $0.1 \times 10^{9}$ cells $\cdot \mathrm{L}^{-1}$. A value $\geqslant 0.3 \times 10^{9}$ cells $\cdot \mathrm{L}^{-1}$ was considered elevated [12].

\section{Statistics and study design}

Population- and disease-associated characteristics were assessed for each examination separately. Thereafter, the controlled versus uncontrolled asthma, exacerbations, prevalence of ICS use (among current asthmatics) and smoking prevalence, BMI and lung function (in the whole group) were compared between the sexes. Categorical variables were analysed with chi-squared tests and continuous variables with t-tests.

The longitudinal associations were assessed with a generalised estimating equation (GEE), with both asthma control and lung function measured at the three examinations (8, 16 and 24 years). The overall associations taking all three timepoints into account, as well as the age-specific (cross-sectional) associations between asthma control and lung function at each timepoint, were assessed. Thereafter, the associations were examined in the same model adjusted for sex [20], BMI group [30], allergic sensitisation [31], ICS use [18], current asthma [6], participant's own smoking [11] and environmental smoke exposure [6], measured at each examination, as potential confounders based on previous literature.

Both the unadjusted and adjusted GEE models were stratified for sex. The unadjusted GEE models were

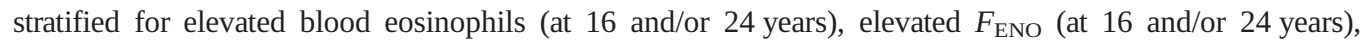
allergic sensitisation (at 8, 16 and/or 24 years) and ever/never smoker, and the overall associations assessed.

As a sensitivity analysis to evaluate the effect of asthma symptom control and lung function not mediated through asthma exacerbations, the GEE model (unadjusted) was performed, excluding data from participants with a reported asthma attack at the given follow-up.

A p-value $<0.05$ was considered statistically significant. 
The statistical analyses were performed with STATA Statistical Software: Release 16 (College Station, TX, USA).

\section{Ethical permission}

The BAMSE study is approved by the Ethical Review Board at Karolinska Institute Dnr: 02-420; Dnr: 2010/1474-31/3; Dnr: 2016/1380-31/2. All caregivers (8, 16 years) and participants (16, 24 years) provided an informed consent.

\section{Results}

\section{Study population}

The included population, with at least one completed spirometry and fulfilling the criteria for asthma at one or more examinations at 8, 16 or 24 years, consisted of 668 individuals (figure 1). In the study group, 541 individuals completed the questionnaire at all three visits (for details on distribution see Supplementary Figure 1). In the group, 53\% were females. A large part of the included group did not persistently fulfil the criteria for asthma; $27 \%$ had asthma at all examinations in which they participated. The study group is presented in detail in table 1.

Uncontrolled asthma was present in 32\% of the participants at 8 and 16 years of age, and 38\% at 24 years of age (table 2).

To assess differences in asthma morbidity, risk factors and medication between the sexes, the group with current asthma at the given follow-up was analysed for differences between females and males with respect to uncontrolled asthma, asthma exacerbations, ICS use and lung function. The prevalence of uncontrolled asthma shifted from being higher among males at 8 years to being higher among females at 24 years, and

\section{TABLE 1 Population characteristics at the three examinations}

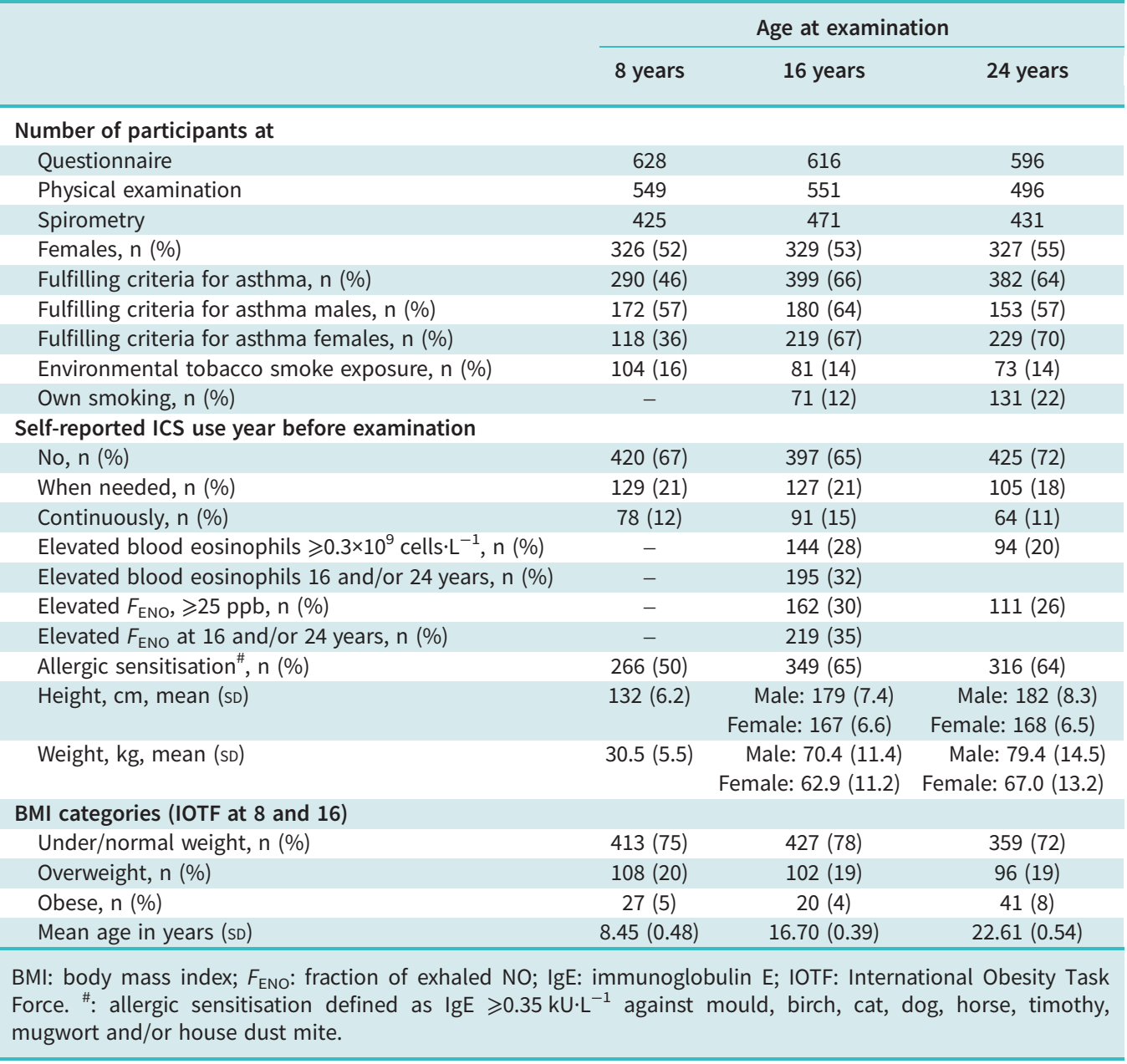


TABLE 2 Asthma control and lung function at the three examinations

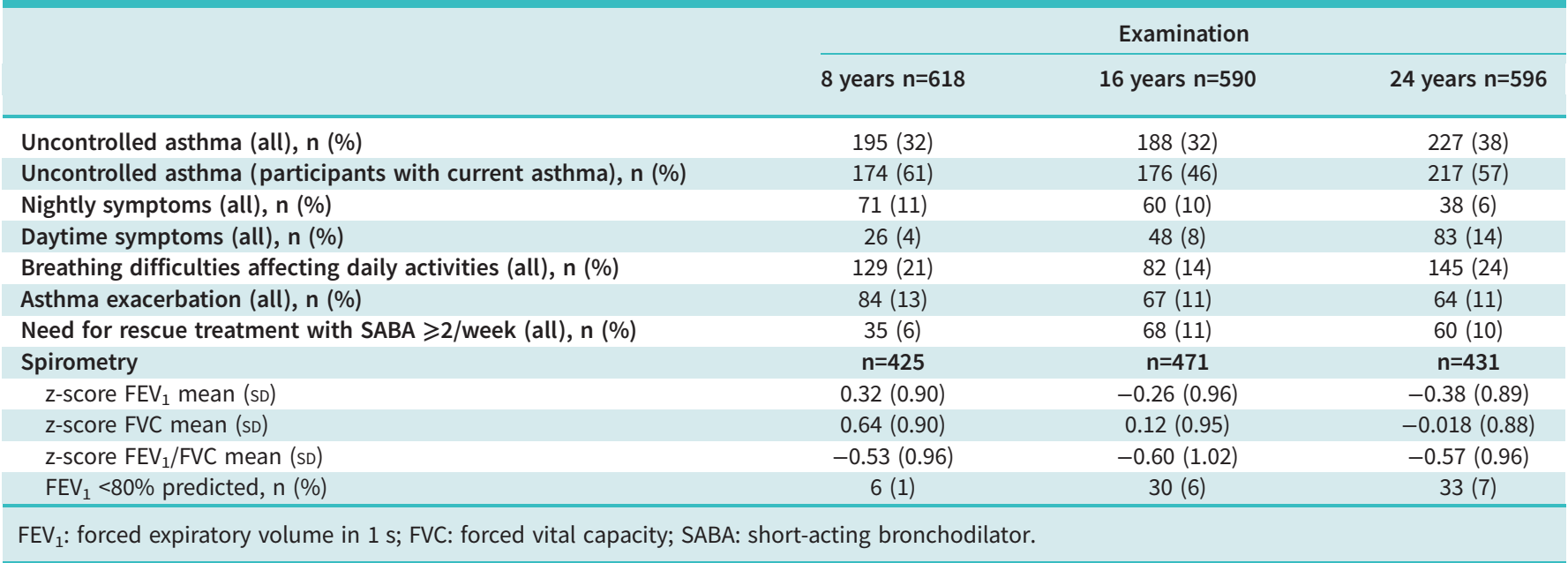

there was a borderline significance for more exacerbations among females ( $18 \%$ versus $12 \%, \mathrm{p}=0.083)$ at

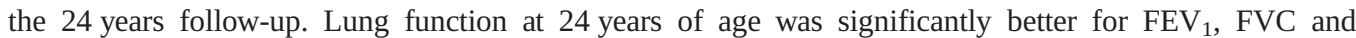
$\mathrm{FEV}_{1} / \mathrm{FVC}$ measured as z-score among the females than among the males (Table 2a- $\mathrm{d}$ of the Supplementary information). There was no difference in BMI or smoking prevalence between the sexes.

Associations between asthma control and lung function

In the overall analysis, uncontrolled asthma was associated with a lower $\mathrm{FEV}_{1} / \mathrm{FVC}$ z-score in both the unadjusted and the adjusted analysis (figure 2).

In the unadjusted age-specific analyses at each examination, uncontrolled asthma was associated with lower $\mathrm{FEV}_{1}$ at 16 years and with lower $\mathrm{FEV}_{1} / \mathrm{FVC}$ at 16 and 24 years, but these associations were not significant after adjustments (figure $3 \mathrm{a}-\mathrm{c}$ ).

In the sex-stratified analyses, the significant overall association between uncontrolled asthma and a lower $\mathrm{FEV}_{1} / \mathrm{FVC}$ was found among females, but not males (figure 2); however this did not represent a significant interaction ( $p_{\text {interaction }}=0.10$ ). In the age-specific analyses, the association between uncontrolled asthma and lower $\mathrm{FEV}_{1} / \mathrm{FVC}$ was found among the females at 24 years in the adjusted analyses (figure 3a-c, unadjusted results presented in Table 3 of the Supplementary information).

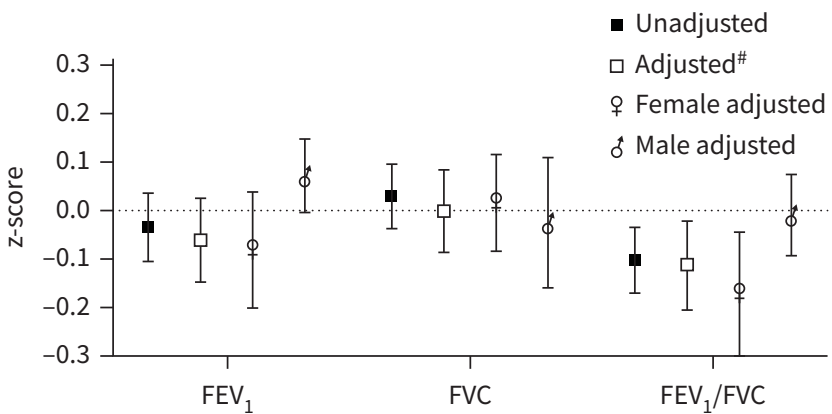

FIGURE 2 Overall estimates (regression coefficient and 95\% Cl): differences in lung function $z$-score for uncontrolled asthma in comparison to controlled asthma from 8 to 24 years of age. \#: adjusted for sex, BMI group, allergic sensitisation, ICS use, current asthma, participant's own smoking and environmental smoke exposure. BMI: body mass index; ICS: inhaled corticosteroid. 

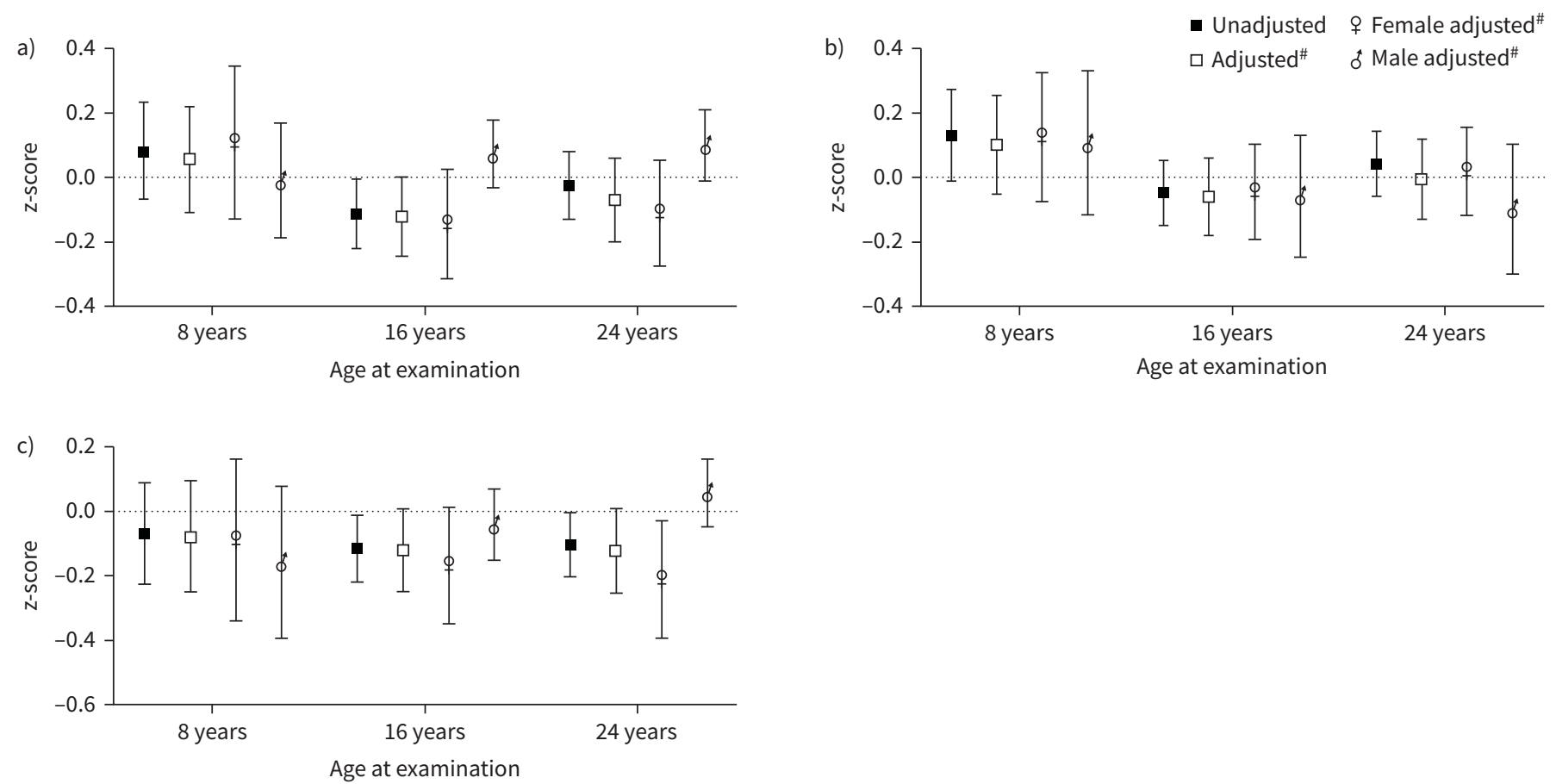

FIGURE 3 Estimates (regression coefficient and 95\% Cl): for the association between uncontrolled asthma and lung function z-score compared to controlled asthma at 8, 16 and 24 years of age. a) $\mathrm{FEV}_{1}$; b) FVC; c) FEV 1 /FVC. "\#: adjusted for sex, BMI group, allergic sensitisation, ICS use, current

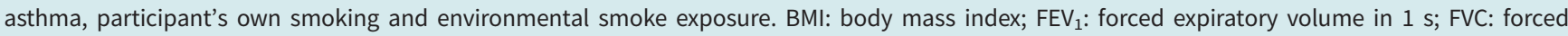
vital capacity; ICS: inhaled corticosteroid.

\section{Analyses stratified by clinical biomarkers and smoking}

In the stratified analysis for elevated blood eosinophils, no clear differences were found in the association between uncontrolled asthma and lung function impairment between the group with elevated and nonelevated blood eosinophils ( $p_{\text {interaction }}>0.5$ ) (figure $4 a$ ).

In the group with elevated $F_{\text {ENO }}$ there was a significant association between uncontrolled asthma and lower $\mathrm{FEV}_{1}$ and $\mathrm{FEV}_{1} / \mathrm{FVC}$ in the overall analysis, which is in contrast to the group with nonelevated $F_{\mathrm{ENO}}$ $\left(\mathrm{FEV}_{1} \mathrm{P}_{\text {interaction }}=0.028, \mathrm{FEV}_{1} / \mathrm{FVC} \mathrm{p}_{\text {interaction }}=0.060\right)$ (figure $4 \mathrm{~b}$ ).

The group with allergic sensitisation had a pattern with more pronounced association between uncontrolled asthma and lower $\mathrm{FEV}_{1}$, although not a significant interaction ( $\mathrm{p}_{\text {interaction }}=0.088$ ) (figure 4c).

The association between uncontrolled asthma and lung function in ever smokers versus never smokers did not show any major differences in the estimates ( $p_{\text {interaction }}>0.2$ ), although the association between uncontrolled asthma and lower $\mathrm{FEV}_{1} / \mathrm{FVC}$ was significant in the never smoking group only (figure 4d).

\section{Sensitivity analysis}

The sensitivity analyses for uncontrolled asthma excluding the individuals reporting an asthma attack the previous year yielded a significant association between uncontrolled asthma and a lower $\mathrm{FEV}_{1} / \mathrm{FVC}$ ratio compared to the group with controlled asthma (z-score $-0.082 ; 95 \%$ CI -0.158 to -0.007 ; $=0.032$ ) (Table 4 of the Supplementary information).

\section{Discussion}

In this investigation, using longitudinal data from a prospective birth cohort, the aim was to evaluate the associations between uncontrolled asthma and lung function measured with dynamic spirometry from 8 to 24 years of age. Our main findings partly confirm our hypothesis, and we found a significant association between uncontrolled asthma and a lower $\mathrm{FEV}_{1} / \mathrm{FVC}$ ratio (z-score) from 8 to 24 years in comparison to the group with controlled asthma. However, this was after stratification for sex primarily found among the females. Further, among the participants with elevated $F_{\text {ENO }}$ an association between uncontrolled asthma and lower $\mathrm{FEV}_{1}$ and $\mathrm{FEV}_{1} / \mathrm{FVC}$ was present, in contrast to participants with normal $F_{\mathrm{ENO}}$. 

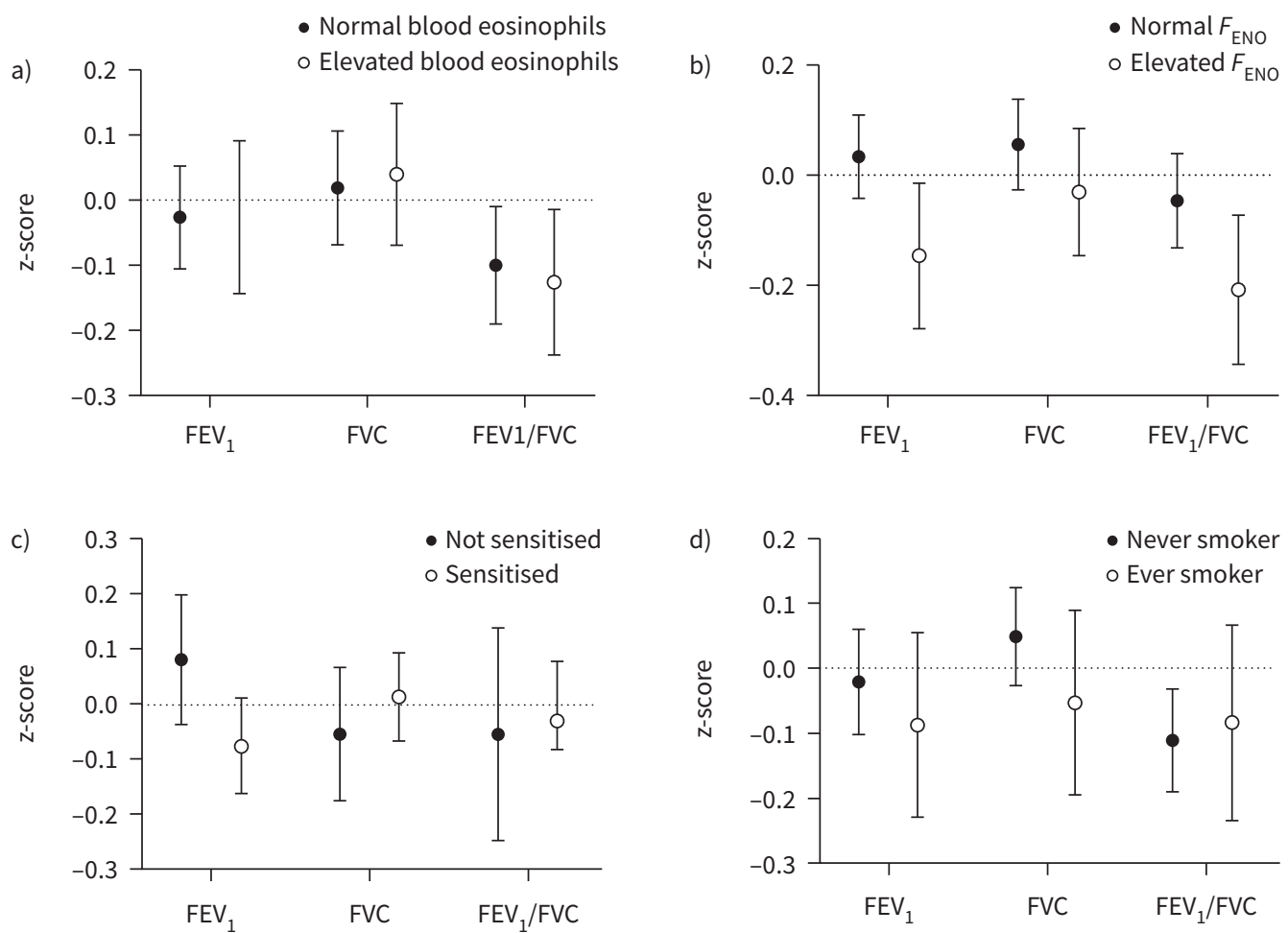

FIGURE 4 a) Association between uncontrolled asthma and lung function measured as z-score (regression coefficient and $95 \% \mathrm{Cl}$ ), compared to controlled asthma, stratified for elevated blood eosinophils (B-Eos) $\geqslant 0.3 \times 10^{9}$ cells $\mathrm{L}^{-1}$. b) Association between uncontrolled asthma and lung function measured as $\mathrm{z}$-score (regression coefficient and $95 \% \mathrm{Cl}$ ), compared to controlled asthma, stratified for elevated fraction of exhaled nitric oxide $\left(F_{\mathrm{ENO}}\right) \geqslant 25 \mathrm{ppb}$. c) Association between uncontrolled asthma and lung function measured as z-score (regression coefficient and $95 \% \mathrm{Cl}$ ), compared to controlled asthma, stratified for allergic sensitisation (positive Phadiatop) at any examination. d) Association between uncontrolled asthma and lung function measured as z-score (regression coefficient and $95 \% \mathrm{Cl}$ ), compared to controlled asthma, stratified for never or ever smoking.

Asthma exacerbations have previously been found to be associated with impaired lung function development and airflow obstruction [16, 32]. Although uncontrolled asthma has been found to be a risk factor for lower maximally attained lung function [11], and bronchial hyper-responsiveness has been found to be associated with reduced lung function [17, 33], no studies to our knowledge have directly addressed the association between asthma control and lung function from childhood to early adulthood and the impact of sex.

An unexpected finding was the pronounced sex difference in the association between uncontrolled asthma and airflow obstruction, found primarily among females. The female-to-male ratio in current asthma in the group increased over the investigated time period, in line with the literature [20]. However, this was adjusted for in the main analysis. The males also had a generally poorer lung function (measured as z-score) than the females at 24 years of age, possibly indicating other factors to be more important for lung function among asthmatic males than females. We found a nonsignificant trend for more prevalent uncontrolled asthma and more exacerbations in females than in males with increasing age, while the self-reported use of ICS was similar between the groups. This could possibly indicate an undertreatment, or inefficient treatment, among the participating females. In a study by DiJkstra et al. [34], ICS was (in adults) found to reduce the decline of $\mathrm{FEV}_{1}$ in males with asthma while this was not found in women, despite a similar rate of decline before the onset of ICS treatment. Similarly, Convery et al. [35] found a poorer steroid response in airway responsiveness among women than men. The previously described risk during adolescence, of "getting lost" between caregivers in the transition from paediatric to adult healthcare could further lead to a delay in diagnosing new-onset asthma, more prevalent among females during adolescence. This may also result in lack of initiation or poor adherence to maintenance therapy with potentially severe side-effects such as asthma attacks and uncontrolled asthma. The finding that an 
elevated $F_{\text {ENO }}$ was a risk factor for an enhanced association between asthma control and airflow obstruction could support this hypothesis, taking into consideration $F_{\text {ENO }}$ as a marker for steroid-sensitive asthma [36]. Sex hormones [37] could be another factor at play affecting females and males differently, although in females, uncontrolled asthma seems to affect lung function more than in males.

Elevated $F_{\text {ENO }}$ was found to affect the association between uncontrolled asthma and lower lung function, making it more pronounced. In contrast, the association between uncontrolled asthma and lung function was not affected by elevated blood eosinophil levels despite higher blood eosinophils previously having been linked to impaired lung function development in adolescence and early adulthood [11].

Blood eosinophils and $F_{\text {ENO }}$ are both markers for type-2 inflammation. $F_{\text {ENO }}$ is increased by type-2 inflammation in the airway epithelium [38], while the eosinophil level in the blood implicates a systemic component of the inflammation. A possible interpretation of our results is that poor asthma control with elevated $F_{\text {ENO }}$ is a sign of local asthmatic airway inflammation, leading to airway injuries and development of airflow obstruction. The blood eosinophil level, on the other hand, is in itself associated with airflow obstruction, also shown in nonasthmatic populations [13,39], and the effect is possibly less dependent on asthma control.

In this study a well characterised group of young adults followed from school age was investigated with spirometry and information on allergic sensitisation and biomarkers for inflammation. The group population was large, and the data were collected in a structured manner by experienced personnel. However, there are some limitations to take into consideration when interpreting the results. Only participants with asthma at any of the investigated time points were included, and the included group is not representative of the BAMSE cohort as a whole, or of the general population. This was done to avoid the confounding from asthma in a population-based sample, but still cover also "asthma susceptible" participants with previous or incidental asthma during adolescence and early adulthood. To compensate for the shifting presence of asthma, the main analysis and the gender-stratified analyses were adjusted for current asthma.

Pre-bronchodilatory spirometry measurements were used, and uncontrolled asthma at the examination could give falsely low values. This is a limitation that could overestimate the association with lower lung function.

\section{Conclusion}

Uncontrolled asthma is associated with lower $\mathrm{FEV}_{1} / \mathrm{FVC}$ during childhood and early adulthood and is affected by $F_{\text {ENO }}$ levels and sex. An intensified treatment of children and adolescents with symptomatic asthma and especially with elevated $F_{\text {ENO }}$ could have important implications for future lung health. Our results further suggest females with uncontrolled asthma to be at a higher risk for lung function impairment and airflow obstruction than females with controlled asthma, in contrast to males where this association was not found, despite a generally worse lung function than the females at 24 years.

The sex difference in the association between asthma control and lung function, despite similar background characteristics found in our study, implicates a need for further research.

Acknowledgements: We thank the children and parents participating in the BAMSE cohort and all staff involved in the study over the years.

Provenance: Submitted article, peer reviewed.

Conflict of interest: I. Mogensen has nothing to disclose. J. Hallberg reports lecture fees from AstraZeneca $A B$ outside the submitted work. S. Ekström has nothing to disclose. A. Bergström has nothing to disclose. E. Melén reports advisory board reimbursement from AstraZeneca, Chiesi, Novartis and Sanofi outside the submitted work. I. Kull reports grants from the Swedish Research Council and the Swedish Heart Lung Foundation for the present manuscript.

Support statement: This study was supported by grants from the Swedish Research Council, the Swedish Research Council for Health, Working Life and Welfare, Formas, the Asthma and Allergy Association, the Swedish Heart Lung Foundation, the European Research Council (TRIBAL, grant agreement 757919), the Swedish Asthma and Allergy Research Foundation, and Region Stockholm (ALF project, and for cohort and database maintenance). Funding information for this article has been deposited with the Crossref Funder Registry. 
References

1 Lozano R, Naghavi M, Foreman K, et al. Global and regional mortality from 235 causes of death for 20 age groups in 1990 and 2010: a systematic analysis for the Global Burden of Disease Study 2010. Lancet 2012; 380: 2095-2128.

2 Agusti A, Faner R. Lung function trajectories in health and disease. Lancet Respir Med 2019; 7: 358-364.

3 Wang G, Hallberg J, Um Bergström P, et al. Assessment of chronic bronchitis and risk factors in young adults: results from BAMSE. Eur Respir J 2020; 55: 2000544.

4 Stanojevic S, Wade A, Stocks J, et al. Reference ranges for spirometry across all ages. Am J Respir Crit Care Med 2008; 177: 253-260.

5 Berry CE, Billheimer D, Jenkins IC, et al. A distinct low lung function trajectory from childhood to the fourth decade of life. Am J Respir Crit Care Med 2016; 194: 607-612.

6 Bui DS, Lodge CJ, Burgess JA, et al. Childhood predictors of lung function trajectories and future COPD risk: a prospective cohort study from the first to the sixth decade of life. Lancet Respir Med 2018; 6: 535-544.

7 McGeachie MJ, Yates KP, Zhou X, et al. Patterns of growth and decline in lung function in persistent childhood asthma. N Engl J Med 2016; 374: 1842-1852.

8 Agertoft L, Pedersen S. Effects of long-term treatment with an inhaled corticosteroid on growth and pulmonary function in asthmatic children. Respir Med 1994; 88: 373-381.

9 Ödling M, Andersson N, Hallberg J, et al. A gap between asthma guidelines and management for adolescents and young adults. J Allergy Clin Immunol Pract 2020; 8: 3056-3065.e3052.

10 Ödling $\mathrm{M}$, Jonsson $\mathrm{M}$, Janson $\mathrm{C}$, et al. Lost in the transition from pediatric to adult healthcare? Experiences of young adults with severe asthma. JAsthma 2020; 57: 1119-1127.

11 Wang X, Mensinga TT, Schouten JP, et al. Determinants of maximally attained level of pulmonary function. Am J Respir Crit Care Med 2004; 169: 941-949.

12 Mogensen I, Vonk JM, Wijnant SRA, et al. Blood eosinophil level and lung function trajectories: cross-sectional and longitudinal studies in European cohorts. ERJ Open Res 2020; 6: 00320-02020.

13 Hancox RJ, Pavord ID, Sears MR. Associations between blood eosinophils and decline in lung function among adults with and without asthma. Eur Respir J 2018; 51: 1702536.

14 Mogensen I, Alving $\mathrm{K}$, Dahlen SE, et al. Fixed airflow obstruction relates to eosinophil activation in asthmatics. Clin Exp Allergy 2019; 49: 155-162.

15 Bai TR, Vonk JM, Postma DS, et al. Severe exacerbations predict excess lung function decline in asthma. Eur Respir J 2007; 30: 452-456.

16 O'Byrne PM, Pedersen S, Lamm CJ, et al. Severe exacerbations and decline in lung function in asthma. Am J Respir Crit Care Med 2009; 179: 19-24.

17 Grol MH, Gerritsen J, Vonk JM, et al. Risk factors for growth and decline of lung function in asthmatic individuals up to age 42 years. Am J Respir Crit Care Med 1999; 160: 1830-1837.

18 O'Byrne P, Fabbri LM, Pavord ID, et al. Asthma progression and mortality: the role of inhaled corticosteroids. Eur Respir J 2019; 54: 1900491.

19 Bloom Cl, Nissen F, Douglas IJ, et al. Exacerbation risk and characterisation of the UK's asthma population from infants to old age. Thorax 2018; 73: 313-320.

20 Vink NM, Postma DS, Schouten JP, et al. Gender differences in asthma development and remission during transition through puberty: the TRacking Adolescents' Individual Lives Survey (TRAILS) study. J Allergy Clin Immunol 2010; 126: 498-504.e491-496.

21 Almqvist C, Worm M, Leynaert B. Impact of gender on asthma in childhood and adolescence: a GA2LEN review. Allergy 2008; 63: 47-57.

22 Wickman M, Kull I, Pershagen G, et al. The BAMSE project: presentation of a prospective longitudinal birth cohort study. Pediatr Allergy Immunol 2002; 13: 11-13.

23 Melén E, Bergström A, Kull I, et al. Male sex is strongly associated with IgE-sensitization to airborne but not food allergens: results up to age 24 years from the BAMSE birth cohort. Clin Transl Allergy 2020; 10: 15.

24 Hohmann C, Pinart M, Tischer C, et al. The development of the MeDALL Core Questionnaires for a harmonized follow-up assessment of eleven European birth cohorts on asthma and allergies. Int Archives Allergy Immunol 2014; 163: 215-224.

25 Global Initiative for Asthma (GINA). A Pocket Guide for Health Professionals. https://ginasthma.org/ wp-content/uploads/2020/04/Main-pocket-guide_2020_04_03-final-wms.pdf, Date last updated: 2020. Date last accessed: 20210115.

26 Cole TJ, Lobstein T. Extended international (IOTF) body mass index cut-offs for thinness, overweight and obesity. Pediatr Obes 2012; 7: 284-294.

27 Quanjer PH, Stanojevic S, Cole TJ, et al. Multi-ethnic reference values for spirometry for the 3-95-yr age range: the global lung function 2012 equations. Eur Respir J 2012; 40: 1324-1343.

28 ATS/ERS recommendations for standardized procedures for the online and offline measurement of exhaled lower respiratory nitric oxide and nasal nitric oxide, 2005. Am J Respir Crit Care Med 2005; 171: 912-930. 

studies. Lancet Respir Med 2018; 6: 526-534.

32 Calhoun WJ, Haselkorn T, Miller DP, et al. Asthma exacerbations and lung function in patients with severe or difficult-to-treat asthma. J Allergy Clin Immunol 2015; 136: 1125-1127.e1124.

33 Rijcken B, Schouten JP, Xu X, et al. Airway hyperresponsiveness to histamine associated with accelerated decline in FEV1. Am J Respir Crit Care Med. 1995; 151: 1377-1382.

34 Dijkstra A, Vonk JM, Jongepier $\mathrm{H}$, et al. Lung function decline in asthma: association with inhaled corticosteroids, smoking and sex. Thorax 2006; 61: 105-110.

35 Convery RP, Leitch DN, Bromly C, et al. Effect of inhaled fluticasone propionate on airway responsiveness in treatment-naive individuals - a lesser benefit in females. Eur Respir J 2000; 15: 19-24.

36 Smith AD, Cowan JO, Brassett KP, et al. Exhaled nitric oxide: a predictor of steroid response. Am J Respir Crit Care Med 2005; 172: 453-459.

37 Macsali F, Svanes C, Bjørge L, et al. Respiratory health in women: from menarche to menopause. Expert Rev Respir Med 2012; 6: 187-200; quiz 201-202.

38 Alving K, Malinovschi A. Basic aspects of exhaled nitric oxide. Eur Respir Monograph 2010; 49 : 1-31.

39 Shapira U, Krubiner M, Ehrenwald M, et al. Eosinophil levels predict lung function deterioration in apparently healthy individuals. Int J Chron Obstruct Pulmon Dis 2019; 14: 597-603. 